\title{
Raising the ethical standards in neurosurgery
}

\author{
Daniel K. Sokol • Samantha Hettige
}

Received: 16 June 2009 / Accepted: 16 June 2009 / Published online: 9 July 2009

(C) Springer-Verlag 2009

The historically aware neurosurgeon cannot but be astounded by the remarkable progress of the specialty since the early 1900s. Around that time, the neurologist David Ferrier described neurosurgery as 'a sort of polite way of committing suicide' [1]. Today, spectacular neuroimaging techniques, neuroanaesthesia, neurostimulation and brain-computer interfaces, the prospect of neural transplantation using stem cells, and advances in operating microscopes, surgical instruments and technique are only some of the developments that reflect the evolution of the specialty.

Progress in medicine, however, often presents new ethical challenges, and neurosurgery is no exception. Our ability to manipulate and interfere with the brain, and the implications of such an ability on personal identity, consciousness and wellbeing, have raised such an array of ethical issues that a new branch of bioethics has emerged to address them: neuroethics $[4,9]$. While we can open the closed box of the skull with greater ease and safety than ever before, it is not infrequent for neurosurgeons to approach an operation, be it an awkwardly placed petroclival meningioma, a large infiltrating highgrade glioma or an unpredictable cerebral aneurysm, knowing that there is a chance that their patients may die

D. K. Sokol $(\bowtie)$

Lecturer in Medical Ethics and Law, St George's,

University of London,

Cranmer Terrace,

London SW17 0RE, United Kingdom

e-mail: daniel.sokol@talk21.com

\section{S. Hettige}

Department of Neurosurgery, King's College Hospital,

Denmark Hill,

London SE5 9RS, United Kingdom

e-mail: samanthahettige@hotmail.com or be so cognitively impaired as a result of the operation or its complications that they may no longer be able to make decisions.

The invasiveness of many intracranial operations and the risks of neurological deficit distinguish neurosurgery from other specialties. It seems sensible, given these features, that neurosurgeons not only inform patients of the possible outcomes of high-risk operations, but also offer competent patients the possibility to discuss what they would like to happen if they lose the ability to make decisions ("some patients in your situation have views on what they would want if their condition deteriorates after the operation. The chances of this happening are low, but some patients appreciate the opportunity to make plans. Do you have any views on this?") [3].

Depending on the patient's answer, the surgeon could mention the possibility of creating a Durable Power of Attorney (or, in the UK, a Lasting Power of Attorney), which would allow patients to appoint a person (or several persons) to make decisions on their behalf if unable to do so themselves. Alternatively, patients may want to make an advance directive (formally called an 'advance decision' in the UK), specifying what treatment they would not want to receive in the future. If the patient has preferences but does not wish to record them in such a formal manner, the surgeon can document them in the notes. This will provide some indication of the patient's views and wishes that may be of future use. It is important to raise the subject in this preoperative encounter (rather than wait to see how the situation evolves) for it may be the last time the surgeon sees the patient when he or she still has capacity.

If patients accept the offer to have this discussion (and doubtless some patients would rather not), it will allow clinicians to continue respecting their autonomy when they 
are no longer autonomous and, in cases where withholding or withdrawing life-sustaining treatment is an option, will reduce the difficult, often agonising 'best interests' decisions at life's end. It will also reduce the likelihood of conflict between relatives and the clinical team, as well as disagreements within the multidisciplinary team itself, with all the emotional stress, complaints and legal challenges that such disputes can generate.

One of the authors (DKS) has worked in a hospital in Portland, Oregon (USA) where clinicians were encouraged to use the acronym PARQA when obtaining consent from patients. $\mathrm{P}$ is for 'procedure' (explain the procedure), $\mathrm{A}$ is for 'alternatives' (explain the alternatives), $\mathrm{R}$ is for 'risks' (explain the risks and benefits of the procedure and its alternatives), $\mathrm{Q}$ is for 'questions' (invite the patient to ask questions) and, for higher risk procedures, $\mathrm{A}$ is for 'advance care planning'. The neurosurgeon would write PARQA in the patient notes as evidence that he or she had been through the consent process.

Although advance care planning is relatively common in some institutions in North America, it remains rare in most parts of the world. We expect that this proposal for advance care planning will sit uneasily in the minds of some neurosurgeons. Why dwell on low probability outcomes and run the risk of upsetting the already frightened patient? We do not suggest that neurosurgeons have this conversation with all patients. It may be inappropriate in some situations. When to raise the issue will be a matter of judgement, as it is in so many areas of neurosurgery. Just as surgeons will assess aneurysm size, age of the patient, concurrent medical conditions, previous history of aneurysms and several other factors in deciding whether to operate on an unruptured intracranial aneurysm, so should they assess the severity of the condition, the nature and likelihood of complications, the informational preferences of the patient, the cognitive ability and emotional stability of the patient, and other factors in determining whether it is appropriate to broach the topic of advance care planning with the patient. In some circumstances, it may not be practicable for reasons of time and clinical urgency to discuss this, nor to obtain more than a truncated form of consent.

In discussing high-risk operations with patients, it is tempting for surgeons to mention the risks of death and serious morbidity, and swiftly retreat from that gloomy area as soon as enough information has been disclosed to satisfy the demands of informed consent. While maintaining a patient's hope is important, the line between hope and false hope is thin [8]. Overinflated hope and extravagant expectations can invalidate consent. Do such patients know sufficiently what they are signing up for? And there is the ever-present danger of self-deception, where the surgeon's belief that his actions benefit the patient disguises the real reason behind the act, namely that it protects the surgeon from a difficult, unpleasant, tearful or time-consuming discussion. It is widely acknowledged that while some surgeons are open to the mortal dangers of their art, others avoid the mere thought of death or serious morbidity, although we suspect that a small minority of neurosurgeons fall within this last category [3].

Far from frightening the patient and dashing hope, we believe an advance care planning discussion, sensitively conducted, may reassure the patient. The patient may have had the 'what if it goes wrong?' thought in mind but felt unable to raise the issue. This can foster anxiety and a feeling of isolation. The power imbalance between surgeon and patient, especially in a high-stakes area like neurosurgery, can hardly be overstated [2]. Offering patients the opportunity to discuss future care can provide them with a sense of control and demonstrates a respect for them as persons, as individuals with distinct values and preferences. The relationship between surgeon and patient can emerge strengthened from the discussion. It also serves to calibrate patients' expectations with the reality of the situation.

It would be naïve to deny that raising the topic, even if not pursued, may distress patients. Some patients, perhaps of an optimistic bent, may well get upset when confronted with this unwelcome prospect. Yet the possibility of distress, present in all disclosures of bad news from a diagnosis of cancer to the impossibility of cure, is not in itself sufficient to justify avoiding the issue. Such was the reason given to justify the nondisclosure of a cancer diagnosis to patients until 30 years ago in the UK and the US [7]. Distress cannot always be avoided, nor should it be. In a chapter on low-grade gliomas, Henry Marsh notes that 'It must never be forgotten that the diagnosis of a brain tumor is quite devastating for the patient and the patient's family' [6]. There are times when distress is an appropriate response and when a total lack of distress would be of concern. If we are committed to respecting the autonomy of patients, then patients should be informed of the possibility of advance care planning in the event of an adverse outcome. The potential benefits of raising the issue, in our view, clearly outweigh the harms of doing so.

Neurosurgery has never been safer, but entering the once forbidden 'closed box' remains treacherous in many situations. Neurological damage leading to loss of capacity, either as a result of the disease process or the operation itself, is not unusual. It is thankfully rare for neurosurgery to render a competent patient incompetent, but it does occur, and it is far more common for patients undergoing major intracranial surgery to lose capacity in the weeks or months following surgery. We have argued that in at least some of those situations neurosurgeons should initiate a 
discussion with patients about what they would want if they lost the capacity to make decisions. It seems prudent to have that discussion preoperatively even if patients are more likely to lose capacity as a result of the disease process than the operation.

This may seem a bold suggestion, but history shows that neurosurgeons have not shied away from opportunities to improve their art. This one is not of the kind familiar to most neurosurgeons - the technological, technical or therapeutic sort-but an improvement in method and ethics. This proposal supports the view, espoused by Jones, McCullough and Richman, that "ethics in surgery is not an 'ivory tower' enterprise but a deliberately and intensely practical tool designed to improve patient care" [5].

Acknowledgements We thank Professor Tony Bell for helpful comments on an earlier draft.

\section{References}

1. Bliss M (2005) Harvey Cushing; a life in surgery. Oxford University Press, Oxford

2. Brody H (1992) The healer's power. Yale University Press, London

3. Fins J (2006) A palliative ethic of care. Jones and Bartlett Publishers, London

4. Glannon W (2007) Bioethics and the brain. Oxford University Press, Oxford

5. Jones J, McCullough L, Richman B (2008) The ethics of surgical practice. Oxford University Press, Oxford

6. Marsh H (2006) Low-grade gliomas in adults. In: Moore A, Newell D (eds) Tumor neurosurgery. Springer, London, pp 155-166

7. Sokol D (2006) How the doctor's nose has shortened over time; a historical overview of the truth-telling debate in the doctor-patient relationship. J R Soc Med 99:632-636

8. Sokol D (2006) What is false hope? J Clin Ethics 17:367-368

9. Wind J, Anderson D (2008) From prefrontal leukotomy to deep brain stimulation: the historical transformation of psychosurgery and the emergence of neuroethics. Neurosurg Focus 25:E10 